\title{
LA CO-CREATIVIDAD COMO REFLEJO DEL CO-PENSAR Y CO-SENTIR DE LA ETNIA ANCESTRAL LITO-LENGUAJERA PRECOLOMBINA DE SAN AGUSTIN
}

\section{COLLECTIVE CREATIVITY AS COLLECTIVE THINKING AND FEELING PRACTICE'S PRODUCT AMONG COMMUNITY OF LITO-LENGUAJERA ANCESTRAL PRECOLOMBINA ETHNIA IN SAN AGUSTIN}

\author{
José Uriel Leal Zabala*, David Soler Lemus**
}

\begin{abstract}
Resumen
El simbolismo indígena de la cultura prehispánica de los Andes Colombianos -ahora convencionalmente llamada San Agustín- permanece aún con nosotros, que hablamos español como lengua materna y vivimos en un mundo interpretativo tan distinto. Es suficiente considerar que mientras ellos tenían aire y ríos puros, montañas y árboles como contexto, nosotros tenemos ciudades de pavimento, grandes edificios, autos veloces y humo. Nosotros, que fuimos conquistados somos los mismos: siendo también conquistadores estamos en la conquista de nuestro propio ser. Perdimos por un tiempo la huella de un antiguo conocimiento pero nunca perdimos la facultad del lenguaje. Hemos tenido la posibilidad de aprender, reflexionar y enseñar sobre esta comunidad ancestral para así rescatar sus códigos y su sabiduría.
\end{abstract}

Palabras clave: Arte de San Agustín, simbolismo, cultura, mayéutica, identidad, eternidad, mestizajear, lenguaje.

\begin{abstract}
The indigenous symbolism of the pre-hispanik culture of the Colombian Andes -now conventionally called San Agustín- remains with us. We who speak Spanish as mother's language and live in a different interpretative world compared to the pre-hispanik one, whereas her lived in a natural context of rivers, trees an mountains, we have cities of pavement, huge buildings, fast cars and smoke. We, who were conquered, are the same: being also conquerors we are in the conquest of ourselves. We lost the track of an antique knowledge but we have never lost the faculty of language. We have had the advantage to learn, reflect and teach about this ancestral community in order to rescue their codes and wisdom.
\end{abstract}

Keywords: San Agustin Art, symbolism, culture, mayéutica, identity, eternity, crossbreeding/mixed races, language.

Artículo recibido: 20/07/2013 Aprobado: 19/08/2013

* Psicólogo de la Universidad Nacional de Colombia. Especialista en Docencia Universitaria de la Universidad Cooperativa de Colombia. Docente Universidad de Cundinamarca, Seccional Girardot. Email: uritolima@hotmail.com

** Doctor en Filosofía del Instituto Goethe de Alemania. Docente Universidad de Cundinamarca, Seccional Girardot. Email: solerlemus@gmail.com

Revista ENTORNOS. Vol. 26, núm. 2. Universidad Surcolombiana. Vicerrectoría de Investigación y Proyección Social, 2013, pp. 51-57 
Una cultura que abre puertas a la eternidad

Esta práctica pedagógica nos lleva a rastrear códigos prehispánicos y hace parte de un macro proyecto etnoeducativo de la Universidad de Cundinamarca en su programa de la Licenciatura en Educación Básica con Énfasis en Humanidades, Lenguas Castellana e Inglesa. Sus propósitos son conocer directamente y, mediante la metodología del vagamundear mayéutico in situ, el legado cultural de nuestros ancestros, sus haceres cognitivos en lo conceptual, silencioso, emocional y practópico; reflexionar y aprehender sobre dicha comunidad, continuar apropiándonos de una pedagogía de rehumanización; rescatar valores de esta cibernética antigua, construir persona mediante el desarrollo de la interculturalidad, el encuentro consigo mismo y la convivencia creadora de la natura y la cultura.

Emprendimos un maravilloso recorrido 0 andadura prehistórica por la infancia de nuestros hermanos mayores. En virtud de sus intensas y variadas relaciones con la geografía de su contexto, conocieron el poder del rayo, del trueno, del fuego y del agua, de la fructífera madre tierra.

Pasaron a confrontarse con la enfermedad y la muerte y a dejarse asombrar por esa tierra rica en admirables materiales, flora y fauna, que los inspiró para urdir colectivamente finos imaginarios, para formar con mayor profundidad y sabiduría su conciencia, para alcanzar finalmente el conocimiento de valiosos misterios existenciales.

Para nosotros, quienes pertenecemos a la Licenciatura en Educación Básica, no es un pasatiempo, ni un andar errático por el mundo primigenio: es el esfuerzo deliberado por conocer y rescatar la sabiduría de nuestra familia antigua, plasmada durante el trabajo diario y de siglos, en su empeño por expresar lo imperecedero, las distintas luchas de la vida humana y el paso personal que luego conduce al más allá, a entrar en el misterio de la muerte, en ese espacio-tiempo absoluto, en el que ies posible encontrar definitivamente la tranquilidad y la saciedad del espíritu?

Los novicios licenciados, frente al copioso caudal estatuario, en intento de descifrar lo humano, armados de un método arcaico y efectivo, el mayéutico, deben primero activar su espíritu y mirar desde sí mismos, dejar fluir sus intuiciones, realizar sus propias observaciones y di- sertaciones, en silencio, a solas ... hasta que nazca la pregunta escudriñadora.

Las esculturas hablan del ciclo que da composición a la sinfonía de la vida, luego este se transforma en jardín de ceremoniales y rituales, cuyo contenido es el trascender, el borrar las fronteras de los mundos y el entrar a vivir en un suave ritmo de paz.

Este patrimonio mítico-poético ancestral, manifestación de todo un afán de conocimiento, del ideal de perfeccionamiento humano, está al alcance de todos los colombianos y del mundo entero; aprovechémoslo e integrémoslo a nuestra bitácora de vuelo por los senderos de la inmensidad, que nunca es concebible exhaustivamente por la razón humana.

La mitopoética de este prehispánico pueblo es una de las concepciones más geniales producida por nuestra humanidad precolombina. La Fuerza que ordenó este Microcosmos dejó en las entrañas de la tierra una multiplicidad de poderes energéticos, engendró todas las formas existentes en el supramundo e inframundo a través de la simbiosis de los poderes divinos que poseen los Animales con el misterioso poder del hombre y la naturaleza.

Estos pobladores instauraron categorías precisas en sus procesos fúnebres y ritos de paso. Superando el tiempo, los hipogeos megalitos conservan fresca toda la serenidad, solemnidad, equilibrio y alegría con que este pueblo concebía las diadas vida-muerte; hombre-mujer; realidad-otredad; salud-enfermedad; arriba-abajo; lluviatierra; cerrado-abierto; día-noche; natura-cultura.

Toda su milenaria cultura los condujo a preguntarse: ¿Hacia dónde nos dirigimos los seres humanos? Al parecer percibieron que todo lo que existía en su derredor estaba regido por Fuerzas Espirituales Trascendentes y, que esas energías presentaban varias formas, diferentes maneras de ser, múltiples funciones, grados infinitos de aplicación, en suma, aliados para enfrentarse al lado activo del infinito.

Estos nativos escultores ejercían el ocio culto y gracias a una suerte de mecenazgo, gozaban del amparo y manutención total por parte de su comunidad; como élite privilegiada, se dispusieron a leer en la realidad natural su realidad virtual, borrando las fronteras de sus mundos. Todo esto lo hicieron con serenidad, con parsimoniosa calma, asistidos por la energía cósmica, por el poder de 
sus cerebros, por las prodigiosas fuerzas de los dioses hacedores, de la madre tierra.

Sus lito-esculturas nos hacen ver el maravilloso artificio de los símbolos tallados en piedra artística; en ellas se reflejan el conocimiento de la simetría aplicado a la forma, la agudeza en la selección de un material perenne y la sabiduría del contenido. Esta gramática lítica teleológica fue agradable a toda la comunidad indígena, fue dulce compañera en la soledad de estos parajes $y$, entre todas las disciplinas que cultivaban, se configuró como la que más trabajo tiene, proyecta lucimiento y trasciende al hombre; sus temáticas, su composición, nos permiten suponer que había un gran misterio a resolver: el paso integral a otros mundos.

\section{Nuestro método}

El Método Socrático busca que todas las personas beneficiarias del desarrollo humano participen en la producción de conocimiento e invita a quien indaga, a que lo haga a partir de sí, es decir, luego de despojarse de todos los preconceptos que le sean ajenos. Para esto San Agustín es un escenario propicio; allí es posible la meditación, pues parece que se activase el hemisferio intuitivo; cada ser humano se percibe centro que observa y comprende el universo y al hacerlo se lee y conoce a sí mismo.

Por tal motivo quisimos que los estudiantes, con lápiz y papel en mano, construyeran un diario de campo, en el que fueran quedando reflejados los movimientos y sentimientos internos y libres de sus ocurrencias, pensamientos y voluntad surgidos de su relación personal con la obra lítica. Tal ejercicio facilitaría y acrecentaría el intercambio posterior de impresiones e ideas. Algunas de ellas se encuentran escritas aquí.

No fue fácil, puesto que la costumbre general es recurrir a fuentes autorizadas y desconfiar de nuestras propias capacidades. «Además... las piedras no hablan; entablar diálogo con ellas podría parecer gracioso e incluso erizar la piel».

Sin embargo la persistencia en el cumplimiento del compromiso socrático trajo gratas sorpresas:

Comenzamos a fluctuar entre dos mundos separados por «el marcado contraste entre aquel lugar donde reina

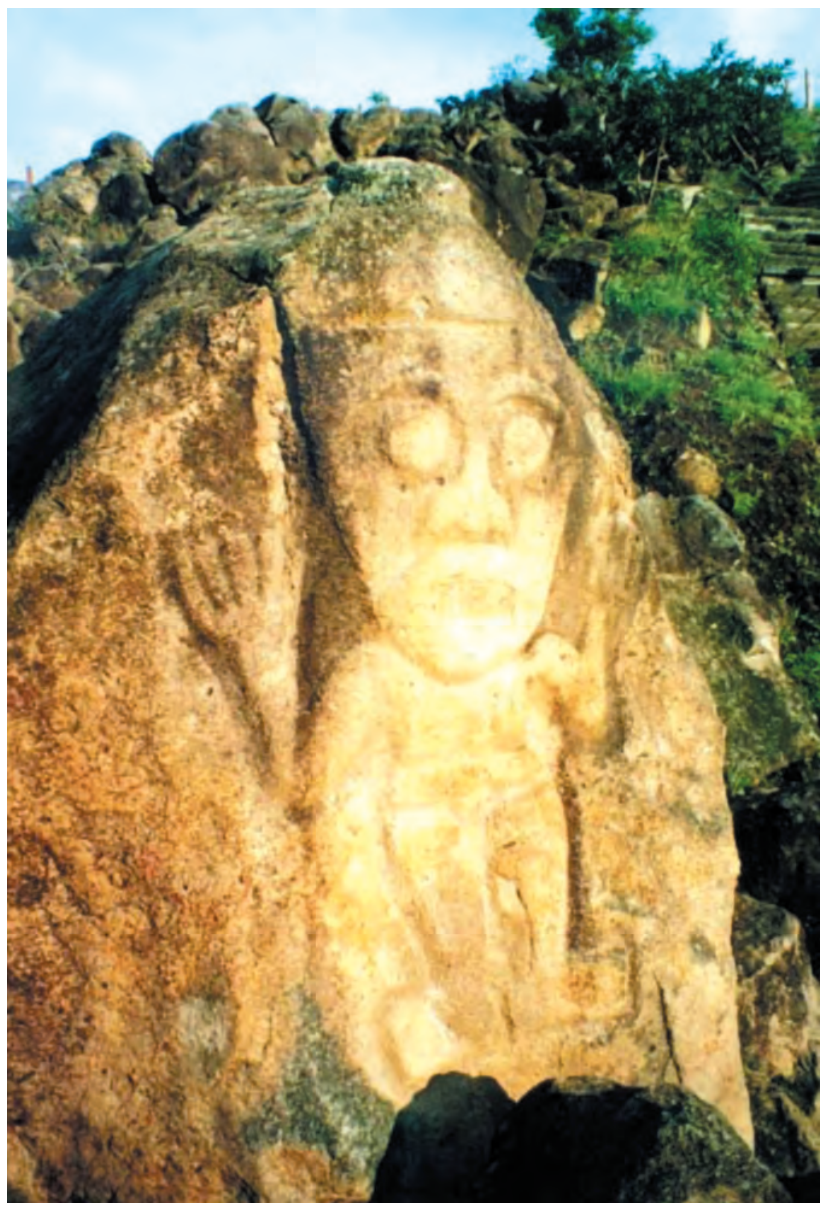

Monumento La Chaquira.

Foto tomada por los autores en San Agustín, Colombia.

la naturaleza y el caótico ambiente de la ciudad, a menudo contaminado y falto de sentido y armonía».

- «Cuando visitamos la cascada fue emocionante, me sentí feliz de estar allí, me sentía libre; el aire, las montañas permitieron deleitar mis sentidos, logré conectarme con el sitio, era casi mágico. La cultura de San Agustín fue privilegiada al vivir en un sitio como éste».

Superando la artificialidad del ambiente turístico que se le ha dado a algunas zonas y la monotonía e insensibilidad en que frecuentemente estamos inmersos, es posible empezar a descubrir la riqueza de un trabajo concentrado, cuidadoso, constante, fino y variado en detalles, realizado con esfuerzo y fatiga, orientado hacia un propósito, que para nosotros, siglos después, plantea numerosas incógnitas:

-«¿Por qué hay guardianes a lado y lado de las tumbas?-

Quizás, si el muerto era hombre su estatua guardiana era 
femenina y viceversa. Así se mantenía la dualidad, entendida como protección y necesidad de otro».

- ¿Qué sentido daban estos pueblos a la muerte? ¿Acaso se va al sol después de morir? ¿Por qué enterrar a sus muertos con vasijas y utensilios, si su conciencia de lo ultraterreno era tan clara?».

-«¿Por qué las diferentes posiciones de los cuerpos en las tumbas contrastan tanto con la forma típica occidental?».

-«¿Serán fantasmas estas piedras? ¿Los muertos vuelven y recobran vida?»

-«Ese diáfano puente, esa transición entre la vida y la muerte en la que insisten los antiguos hacedores de la estatuaria, a veces demuda la risa y la algarabía en silencio y honda reflexión, incluso tristeza y melancolía».

Lo que ellos entendieron para nosotros es oscuro, vemos la muerte y también la vida desde otra perspectiva. Detrás de lo que parece evidente hay otra realidad, pero nuestros ojos son como lentes diseñados por la historia de la colonización.

- «Duele no contar con un abuelo poseedor del saber de aquellas épocas, para aprender del viento que emanase de su boca. De los abuelos de la nuestra en verdad vivenciamos poco. En el presente la juventud se impone ostentosa y majaderamente sobre la vejez, en parte porque muchos hombres y mujeres llegan a ella sin haber aprendido nada, sin tener nada que enseñar para guiar a los que llegan».

Así siguen surgiendo indicios sobre la cosmogonía de este pueblo. En nuestro recorrido por el Parque Arqueológico nos encontramos con una escultura que representa un enorme grano de maíz.

-«!El maíz se ve feliz!» -escribe alguien.

-«Se hace evidente que esta cultura ancestral veía al maíz semejante al sol, constituía para ellos el verdadero Dorado. Como el sol, devolvía las fuerzas al ser cada día».

-«¿Es por ello que todas las estatuas se dirigen hacia él?»

«Cuenta una leyenda», dice nuestro guía, «que el sol lloró una vez, pues le dolió lo que veía hacer a los hombres. Sus lágrimas, al caer, se convirtieron en oro, el oro que más tarde enloquecería a los conquistadores y que se traduce en pesos hoy en día. Hasta que no se devuelvan estas lágrimas al sol no dejará de haber caos y discordia entre los humanos».

Los rasgos zoomorfos presentes en muchas figuras atraen más tarde nuestra atención: - ¿Por qué los dientes puntiagudos de las estatuas?»

- ¿Por qué tienen los ojos la forma de dos águilas que se miran?»

- «Parece que estas gentes admiraban las cualidades de los animales y, al simbolizarlas, las hacían atributos del ser humano». Así es que hallamos al hombre jaguar, al chamán de las metamorfosis.

El guía toca la flauta convocando a reunión a los grupos dispersos. En estos parajes otrora también se tocó este instrumento de viento. Efectivamente se halla una máscara flautista en algún lugar del museo. ¡Observarla lleva cuasi a escuchar su melodía!

Nos reunimos a su son; por cama adoptamos el verde y mullido prado: 360 grados de cielo abierto se ofrecen a nuestros ojos. El guía interpreta ahora músicas andinas.

-«El sonido de la flauta permite que la mente se concentre, ayuda a ver lo que cada uno en ese momento es».

-«Estos son lugares de afluencia musical y de estrellas fluviales».

-«¿De las muchas artesanías que se ofrecen en San Agustín, quién no quiere llevarse a casa una zampoña, una ocarina o un palo de agua?»

¿Cuándo y dónde nacen por vez primera la música, el canto, la expresión, el gesto, la mitopoesía?

- «En cada ser humano que nace se realizan o frustran estos originales y muy significativos prodigios».

- «Dentro de nuestro repertorio musical hay cantos con elementos que vienen de épocas anteriores a la conquista y otros con elementos que vienen de África».

Al encontrar figuras representativas del mambeo de la coca, es fácil entender que los enteógenos permitían a esta cultura el acceso a otros mundos. 
-Uno se pregunta si acaso no causan daños a la salud la ingestión del yahjé, de la hoja de coca, si ellas implican lo que llamamos drogadicción o adicción. Esto lleva a reflexionar sobre todas aquellas plantas que le permiten al ser humano acceder a otros estados de conciencia, acceder a lo inconsciente; también lleva a reflexionar sobre nuestra sociedad y su afán tan distinto, no de comunicarse sino de escapar, mediante la imitación de modelos que toma del cine y de la TV casi sin darse cuenta, e incluso mediante preparaciones tan peligrosas como la cocaína, el alcohol y otras drogas que también pueden alcanzar la categoría de peligrosas como son los tranquilizantes mentales».

-« ¿Somos felices los actuales habitantes de este continente? ¿Había necesidad de crear ciudades de asfalto y gas asfixiante?»

Tal vez si nuestras ciudades se construyeran con sentido, conscientes de unos valores que nos puedan unir ... ¿De dónde hemos tomado el modelo de espacio urbano en que tenemos que vivir? ¿De dónde nuestro vestuario, nuestro modo de adornarnos?

En la dinámica del co-crear, del co-pensar y co-sentir todo lo que hace el ser deriva en un mensaje complejo. Si como grupo académico nos inquietan los procesos de significar, este es un gran ejemplo de integridad en la doble dimensión del signo:

-«A veces en algunas esculturas se hallan colores o vestigios de ellos...»

-«Se observan múltiples formas de vestido y adorno...»

Los artistas descubrieron que la tierra era un ser viviente: En el negro o café oscuro veían el horizonte de capa vegetal, sabían que el útero-entraña (de rojo púrpura) poseía una multiplicidad de poderes generadores. En el blanco o gris claro veían un tercer horizonte de ceniza volcánica. Todo se hizo signo: figuras geométricas, números, posiciones en relación con los astros ${ }^{1}$.

-«Pueden ser también los colores de las serpientes corales, de las plumas de los gallinazos, de las corrientes de aire, según sean cálidas o húmedas».
- «Todo nos lleva a pensar que un amplio sistema simbólico existió entre ellos: Vemos multiplicarse distintos tipos de orejas, ojos y bocas. Encontramos diferentes posiciones y actitudes de los brazos, manos diversas, libres 0 sosteniendo objetos distintos, variantes en los tocados y formas de vestir».

-«¿Qué puedo concluir de esta experiencia? Creo que permitió acercarnos a lo llamado memoria o búsqueda de identidad»».

- «Como humanistas nosotros vemos la cultura de San Agustín por el lado de la construcción y reconstrucción del ser humano; ella contribuye a nuestra formación como seres sociales pertenecientes a un mundo con diferentes modos de pensar, sentir e interiorizar conocimientos particulares y contextuales».

-«Es evidente que esta salida pedagógica fue de gran valor para nosotros futuros docentes; así podemos incrementar nuestros conocimientos acerca de las diferentes culturas, despertar nuestro espíritu creativo, interpretativo y argumentativo. También me parece conveniente resaltar la importancia de ver el mundo con otros ojos, no sólo con lo material sino con los ojos espirituales, con más amor a la vida y sin temor a la muerte».

- «Acepté la invitación que se me hizo, quería rescatar la experiencia ancestral agustiniana, para luego contársela a mi estirpe».

$$
* * *
$$

En la hora crepuscular las estatuas intensifican su quietud, los menores ruidos, el vuelo del búho, la voz del grillo, adquieren un extraño y desconcertante efecto acústico. El alma que ha penetrado en estas piedras ha hablado por sus resquicios.

\section{El mestizajear}

¿Latinoamérica una síntesis sin igual? Parece que ningún otro pueblo reúne en sí tantas razas y culturas diferentes. Tanto en San Agustín como en otros centros de cultura prehispánica, en el continente africano y durante

1. Héctor Llanos Vargas. Los chamanes jaguares de San Agustín, génesis de un pensamiento mitopoético. (Santa Fe de Bogotá: Talleres de Cuatro, 1995). 
la contradictoria y desgarradora expansión de Europa sobre el Nuevo Mundo se echaron las bases fundantes de la cultura latinoamericana, la cual se ha convertido en signo de mestizaje, es decir, en indicador de conflicto, pugna, resistencia, sumisión, mezcla caótica y fraternización entre razas y pueblos, en su lucha por alcanzar un grado más alto de libertad e identidad.

Tanto en la historia del Castellano y del Portugués como en la historia de las Lenguas Amerindias puede descubrirse el testimonio vivo de lo que es el penoso acomodamiento que caracteriza al mestizaje.

En el año 1538-1539 el profesor Francisco Vitoria afirmó desde la Universidad de Salamanca, que los españoles tenían el derecho de entrar al Nuevo Mundo y de participar en la vida social de los indígenas, pero que los habitantes nativos eran los verdaderos señores de esas tierras, en lo público y en lo privado y, que por tanto, podían rechazar autoridad y jurisdicción proveniente de instancias extrañas a ellos y no tenían que someterse a sus juicios ${ }^{2}$. ¡Lástima que entonces esas palabras no se tuvieran en cuenta!

Simón Bolívar había concebido la Independencia de la América Hispana como consecuencia del hecho de que ésta poseía una personalidad que reclamaba un destino propio. »Nosotros somos un pequeño género humano; poseemos un mundo aparte (Carta de Jamaica)».

Roto irremediablemente el orden colonial se quiso implantar sobre sus restos esparcidos y resistentes un orden ideal copiado de Francia, Inglaterra o Estados Unidos. Como tentativa de ruptura y de contradicción era apenas más aventurada que la de los conquistadores de implantar sobre las sociedades indígenas, sobre sus lenguas, sus creencias, sus usos, sus milenarias condiciones, las formas, las normas y los contenidos de la monarquía cristiana de Castilla.

Cuando se intenta, de modo sistemático, reconstruir la historia de las ideas en la América Hispana, desde la Independencia hasta la primera Guerra Mundial, se descubre el más barroco, contradictorio y mezclado panorama difícil de entender.
Los escritores latinoamericanos, en su intento de despejar dicho panorama, han desarrollado una «literatura de enseñanza, de denuncia, de apasionada sed de justicia, a ratos de mesiánica esperanza, pero nunca, en lo más esencial y permanente de su proceso, indiferente a lo colectivo, a lo histórico, a lo popular, a lo político ${ }^{3}$.

Andrés Bello recomienda a los latinoamericanos, aprender a expresar aún mejor su propia personalidad, en una lengua común pero no subordinada; recomienda reemprender la aventura del hombre con una nueva voz y un nuevo aliento.

Durante su proceso de formación, los educadores interesados en aprender a orientar cambios y avances creadores en las vidas y actitudes de las personas y comunidades, deben imaginarse, con la mayor claridad posible, lo que significa trabajar para aunar en una síntesis lo que otros nos ofrecen y nuestra propia originalidad; deben tener presente el sentido que encierra, construir diariamente camino hacia una convivencia humana más rica en contenido, más completa en unidad y más profunda en aspiraciones.

De hecho estamos contribuyendo a generar desde el aula de clase la cultura global o universal que caracterizará al tercer milenio, pero tenemos que buscar y escoger con mayor grado de conciencia los hilos con los que, desde nuestra sociedad, queremos aportar a su tejedura.

El mestizajear evoca nuestra megadiversidad biológi$\mathrm{ca}$, que en nosotros se configura en una megadiversidad etno/tecno/político/social, en la cual radica la complejidad de nuestra articulación histórica y cultural con los ancestros hermanos mayores.

Colombia representa una encrucijada entre lo euroamericano (cuyo centro de gravedad es el Cono Sur), lo afroamericano (el arco de Nueva Orleáns a Bahía, surcando el caribe) y lo indoamericano (Cordillera de los Andes y la cordillera que de Panamá se extiende a México y a California).

Esta complejidad etno-cultural contiene la potencia de una extraordinaria riqueza, pero también anuda el ovi-

2. David Soler Lemus: Die Aneignung der Wirklichkeit als Voraussetzung und Ziel pädagogischen Wirkens. -Schülerdasein in Kolumbien. (Stuttgard: Ibidem-Verlag, 1997)-.

3. Arturo Uslar Pietri: En busca del Nuevo Mundo. (México: Fondo de Cultura Económica, 1969). 
llo de la inmensa dificultad para zurcir una trama que equilibre las distintas expresiones de los pueblos.

No es pues de extrañar, que las aulas escolares en su intimidad alberguen, en las personas de estudiantes y docentes herencias culturales diversas, incluso contrapuestas, procedentes ellas del pasado y de las circunstancias actuales. Como el ser humano no vive únicamente una vida personal, sino también una vida comunitaria -él vive en el mundo-y desea vivir en paz, el aula ha de convertirse en espacio crítico de encuentro, en ambiente civilizado para vivenciar, manejar y disolver conflictos, en zona de aportes y nuevos hallazgos, en centro creador e irradiador de mestizaje cultural.

La interacción dialógica socrática (mestizajear mayéutico) -capaz de activar la facultad del lenguaje, el espíritu crítico, la autodisciplina, la participación democrática, la solidaridad responsable, la imaginación creadora- es fundamental para reinventar la función docente en la universidad y para pensar en una nueva pedagogía, cuyas exigencias son: asumir la complejidad de los problemas sociales, la apertura franca a otras culturas y el cuestionamiento de la propia, superar el mero proceso de enseñanza-aprendizaje lo mismo que una concepción retórica y tecnócrata de educación.

El aprendizaje mayéutico despierta la vocación de verdad, de crítica fundamentada, de posicionamiento argumentado, de fe en sí mismo, de eticidad, admiración y asombro ante un mundo que se devela en su multidimensionalidad universal.

\section{Referencias bibliográficas}

Barber, Benjamin. Strong Democracy. Berkeley y Londres: University of California Press, 1984.

Duque, Luis y otro. Arqueología de San Agustín, Alto de los Ídolos, montículos y tumbas. Bogotá: Lit. Arco, 1979.

Grass, Antonio. Animales mitológicos, diseño precolombino colombiano. Bogotá: Editorial Arco, 1979.

Grass, Antonio. Los rostros del pasado, diseño prehispánico colombiano Bogotá: Editorial Lit. Arco, 1982.

Llanos, Héctor. Los chamanes jaguares de San Agustín, génesis de un pensamiento mitopoético. Santa Fe de Bogotá: Talleres de Cuatro, 1995.

Pietri Uslar, Arturo . En busca del Nuevo Mundo. México: Fondo de Cultura Económica, 1969.

Reichel-Dolmatoff, Gerardo. San Agustín, a culture of Colombia. New York: Editorial Praeger Publishers, 1972.

Soler Lemus, David. Die Aneignung der Wirklichkeit als Voraussetzung und Ziel pädagogischen Wirkens. Stuttgart: Schülerdasein in Kolumbien. Ibidem-Verlag, 1997.

Vasconcelos, José. La raza cósmica. Bogotá: Editorial La Oveja Negra, 1985. 